\title{
Niveles de actividad física y gasto frente a pantallas en escolares: diferencias de edad y género
}

\author{
Levels of physical activity and time spending in front of screens in schoolchildren: differences in age and gender
}

Cristina Andrea Portela-García ${ }^{1,2 *}$ orcid.org/0000-0002-5836-6465

Armando Vidarte-Claros 3 ,4 orcid.org/0000-0002-7982-3848

1. Facultad de Fisioterapia. Universidad de San Buenaventura. Cartagena, Colombia.

2. Grupo de investigación ciencia y movimiento (GIMHUS). Cartagena, Colombia.

3. Departamento de Movimiento humano. Universidad Autónoma de Manizales. Manizales, Colombia.

4. Grupo de investigación Cuerpo - Movimiento. Universidad Autónoma de Manizales. Manizales, Colombia.

\section{Resumen}

Introducción: Cada vez se masifican las tecnologías electrónicas de pantalla entre la población infantil, práctica que ha llevado a un estilo de vida sedentario incrementa los riesgos para la salud de los individuos. Objetivo: Asociar la actividad física y el uso de pantallas, con el índice de masa corporal (IMC) y el género en escolares urbanos de la ciudad de Popayán entre 10 a 12 años. Materiales y métodos: Muestreo aleatorio simple, 440 escolares en edades entre los 10 y 12 años (220 niños/220 niñas). Se realizó la encuesta Physical activity Questionnaire for Older Children-PAQ-C. Estudio prospectivo transversal comparativo, de análisis univariado y bivariado. Resultados: Existe la asociación entre niveles de actividad física moderados y las horas de uso de pantallas $\left(\mathrm{X}^{2}=598,6 ; p=0,00\right)$; así como también entre el nivel de actividad física y el sexo de los escolares $\left(\mathrm{X}^{2}=13,551 ; p=0,004\right)$. Conclusiones: Los niveles de actividad física y el uso de pantallas demuestran que los escolares que usan con mayor frecuencia la televisión, el computador y los videojuegos, despliegan la actividad física en niveles de moderada a baja.

Palabras clave: Niño; actividad física; identidad de género; red de telecomunicaciones; edad escolar; estilo de vida sedentario. (Fuente: DeCS, Bireme).

\begin{abstract}
Introduction: Use of electronic screen technologies are increasingly widespread among children and their frequent use has led to a sedentary lifestyle that increases health risks in this population. Objective: To study the association of physical activity and the use of electronic screen devices with the body mass index (BMI) and gender in urban schoolchildren aged between 10 to 12 years from the city of Popayán (Cauca, Colombia). Materials and methods: A comparative prospective cross-sectional study, with univariate and bivariate analyses, was carried out using a simple random sampling of 440 students between the ages of 10 and 12 years (220 boys/220 girls). The Physical activity Questionnaire for Older Children (PAQ-C) was applied. Results: There is an association between moderate levels of physical activity and hours spent in front of electronic screen devices $\left(\mathrm{X}^{2}=598.6 ; p=0.00\right)$. Likewise, an association between the level of physical activity and gender of schoolchildren was found $\left(\mathrm{X}^{2}=13.551 ; p=0.004\right)$. Conclusions: Children who frequently use television, computers, and/or video games show low to moderate levels of physical activity.
\end{abstract}

Keywords: Child; physical activity; gender identity; telecommunication network; school-age; sedentary lifestyle. (Source: DeCS, Bireme).

\footnotetext{
*Autor de correspondencia

Cristina Andrea Portela García

e-mail: cristinaandrea01@hotmail.com
} 


\section{Introducción}

Los determinantes en salud y entre ellos los estilos y condiciones de vida, juegan un papel importante en el desarrollo de las personas. Según el Ministerio de Salud de Colombia los estilos de vida son "aquellas formas de pensar, sentir y actuar que impactan de manera positiva la salud personal, de los colectivos y del ambiente, las cuales están influenciadas por la historia particular de cada individuo, los grupos sociales y las características económicas, políticas y culturales de cada territorio"(1). Los hábitos de vida sedentarios instaurados desde el colegio y la niñez son una influencia social que se presenta y constituyen un problema de salud pública(2).

En la actualidad la inactividad física de los escolares es cada vez mayor, debido al tiempo dedicado a actividades sedentarias durante su tiempo libre; entre ellas, el uso de pantallas por tiempos prolongados, aumentando así, la prevalencia de obesidad infantil(3), que a su vez se convierte en un factor de riesgo cardio-metabólico.

La Organización Mundial de la Salud (OMS) evidencia la inactividad física como un problema en el ámbito mundial, manifestando que las personas poco activas tienen una probabilidad del $20 \%$ a $30 \%$ adicional de una muerte prematura ${ }^{(4)}$. En las últimas dos décadas la prevalencia de la obesidad infantil está aumentando en todo el mundo, el sobrepeso y obesidad se ha duplicado en los niños de 6 a 11 años de edad y triplicado entre los adolescentes de 12 a 17 años(5,6).

La Encuesta Nacional de Situación Nutricional en Colombia ENSIN, en el año 2015, informó que 7 de cada 100 menores presentan desnutrición crónica, lo cual se considera como un problema de salud pública. Así mismo, el exceso de peso en escolares se incrementó del $18,8 \%$ al $24,4 \%$ en los niños(as) entre 5 y 12 años de edad. Otro dato alarmante es el tiempo que los escolares dedican al uso de las pantallas (televisor-computador) afectando a 7 de cada 10 niños de las áreas urbanas y a 5 de cada 10 de las zonas rurales. Esto dependiendo de la accesibilidad a los dispositivos electrónicos(7).

En un estudio realizado por Martínez et al.,(8) se encontró que los estilos de vida sedentarios repercutieron en la salud mental y física del adolescente, resultando como consecuencia, alteraciones del sistema musculo esquelético, los desbalances posturales y los dolores de espalda, cabeza y cuello, como los más prevalentes en aquellos que permanecieron mucho tiempo inactivos físicamente. Así mismo, se manifestaron variaciones en el comportamiento psicológico del individuo, encontrándose diferencias en el género femenino, caracterizado por presentar en mayor proporción manifestaciones clínicas de dolor relacionadas con el sedentarismo(8).

Borrás y Ugarriza ${ }^{(9)}$ destacan que las posibles causas de la obesidad infantil pueden estar involucradas, además, de los aspectos nutricionales y la genética, los niveles bajos de actividad física, sin dejar de lado el papel fundamental que juegan los padres en el momento de inculcar hábitos y estilos de vida en sus hijos; como por ejemplo dedicar mucho tiempo a la televisión, video juegos y computadores, con la consecuente inactividad física, situación que genera aumento progresivo de peso. La prevención de los factores de riesgo por medio de la modificación de estilos de vida saludables en escolares y el aumento de la práctica de la actividad física, son relevantes en la reducción de actividades sedentarias y por lo tanto disminuyen los riesgos de enfermedades crónicas no transmisibles $(\mathrm{ECNT})^{(9,10)}$.

Estudios científicos realizados en Colombia reflejan la importancia de la actividad física y los beneficios que trae para la salud del individuo(11,12); el sedentarismo, influye negativamente en la salud $y$ en las capacidades físicas de los niños, entre los que se destaca la desnutrición y la reducción del acondicionamiento físico afectando la calidad de $\operatorname{vida}^{(13) .}$

En torno a los niveles de sedentarismo, un estudio realizado muestra que los niños entre 10 y 12 años se encuentran expuestos al uso del computador, los videojuegos y la televisión, sin importar el género. Este estudio relacionó la capacidad cardiorrespiratoria máxima de acuerdo al tiempo de exposición frente a pantallas, evidenciando que los valores más pobres del volumen de oxígeno se relacionan con el género femenino, por lo que se recomendó la práctica de ejercicio físico(14).

Aceptando la evidencia que muestra la globalización con las nuevas tecnologías, se asume en este sentido el gasto de tiempo frente a pantalla como parte del 
estilo de vida de los escolares, por ello, el objetivo del artículo fue asociar la actividad física y el uso de pantallas con el Índice de Masa Corporal (IMC) y el género en escolares urbanos de la ciudad de Popayán entre 10 a 12 años. El cual se deriva del estudio multicéntrico sobre niveles de actividad física realizado en la maestría de Actividad física y deporte de la Universidad Autónoma de Manizales.

\section{Materiales y métodos}

\section{Participantes}

Los participantes fueron 442 escolares de 10 a 12 años de edad, de ambos géneros, ubicados en 12 instituciones educativas en la ciudad de Popayán Colombia, seleccionados de manera aleatoria. Para esta elección se tuvo en cuenta la información obtenida por la Secretaría de Educación del Municipio de Popayán (SEM).

Se diseñó un estudio prospectivo transversal correlacional, empleando para el estudio un muestreo aleatorio estratificado con afijación proporcional con una confiabilidad del 95\%, y un margen de error de $0,3 \mathrm{~kg} / \mathrm{m}^{2}$. Para la estimación de la varianza se tomó como base el estudio "Exposición a pantallas, sobrepeso y desacondicionamiento físico en niños y niñas"(14). La población se organizó por grupos o subpoblaciones (edad y sexo), se homogenizaron los estratos, según la edad la frecuencia fue de 148 niños de 10 y 11 años respectivamente y de 146 escolares de 12 años, por sexo se definió 222 niños y 220 niñas.

\section{Procedimientos}

Se utilizó como técnica la encuesta y como instrumento el formato que daba cuenta de variables sociodemográficas y el instrumento empleado para registrar la actividad física fue el cuestionario de estilo de vida Physical Activity Questionnaire for Children (PAQ-C), validado para Colombia por Herazo y Domínguez en 2012(15), logrando una consistencia interna de 0,73 y 0,78 , que se interpreta como un coeficiente de Cronbach aceptable. El PAQ-C es un cuestionario que mide los niveles de actividad física en los últimos 7 días durante el año escolar, indaga sobre las actividades realizadas durante el tiempo libre y el tiempo que el evaluado permanece frente a la pantalla, entre otros. Para calcular la puntuación final se estima la media de las preguntas, donde 1 indica baja actividad física y 5 indica alta actividad física. En el componente de gasto de tiempo frente a pantalla, el niño debía señalar si utilizaba computador, videojuegos y/o televisión, y en caso afirmativo, informar la dedicación en el número de horas al día y cuantos días a la semana(15). Las variables sociodemográficas del estudio fueron la edad y el sexo.

Los criterios de inclusión fueron: que los niños se encontraran en edades entre 10 y 12 años de edad, estuviera en condiciones de contestar las preguntas de la encuesta que se aplicó de manera autodirigida y contar con el permiso del acudiente.

Se realizó una prueba piloto con 30 escolares de la zona rural con el fin de capacitar a los evaluadores estudiantes de octavo semestre de Educación Física y Fisioterapia de la Universidad del Cauca, quienes realizaron la toma de los datos y revisión de la aplicación del instrumento, el cual no se modificó una vez realizados dichos procesos de capacitación.

Se aplicó la encuesta en un tiempo de seis meses en las 12 instituciones educativas. Una vez definido el muestreo y a partir de una lista aleatoria se seleccionaba un estudiante para participar en el estudio, la encuesta se diligenciaba en un espacio definido por la institución.

\section{Análisis estadístico}

Para el registro de la información, se utilizó el software SPPSS 22 licenciado por la Universidad Autónoma de Manizales. Se desarrolló el análisis univariado de las variables categóricas y posteriormente se hizo un análisis bivariado para identificar la asociación entre las variables del estudio, la asociación se determinó a partir del coeficiente de contingencia, prueba de Chi cuadrado $\left(\mathrm{X}^{2}\right)$, y se complementó mostrando un análisis de correspondencia múltiple (ACM), el nivel de significancia tenido en cuenta fue $p<0,05$.

\section{Consideraciones éticas}

Se diligenciaron los consentimientos informados con los padres y/o acudientes y asentimiento informado por parte del escolar, al igual que las cartas de solicitud de permiso a las instituciones educativas para la realización del estudio. El escolar se encontraba en libertad de retirarse del estudio por voluntad propia. Las implicaciones éticas del estudio se rigieron a partir de la Resolución 008430 de 1993, articulo 11, donde se clasificó como riesgo mayor al mínimo ya que se trabajaba con menores de 18 años, estos elementos fueron avalados por el Comité de 
Bioética de la Universidad Autónoma de Manizales acta 062 de 2017.

\section{Resultados}

La frecuencia de la práctica de futbol está más asociada con el género masculino que se destacó por ser más activo en este deporte, 4 niños de cada 10 afirmaron practicar el futbol en frecuencia de 7 veces o más por semana; en contraste con el género femenino, donde 1 niña de cada 10 participan en este juego, o lo practican de 1 a 2 veces por semana $(p<0,001)$. Cabe destacar que el género femenino realiza con mayor frecuencia la actividad física de saltar la cuerda, donde 8 niñas de cada 10 saltan de 3 a 4 veces a la semana $(38,6 \%$ de las niñas), en comparación a la proporción de niños que practican esta actividad (19,8 \% de los niños). Existe una mayor asociación del género femenino con la práctica de saltar la cuerda $(p<0,001)$.

En general se encuentra una asociación estadísticamente significativa entre el género y los niveles y tipo de actividad física; se evidenció en el género masculino un nivel de actividad más alto que en el femenino, donde 3 niños de cada 10 tienen un nivel de actividad física buena y muy buena $(18,5 \%)$ $\mathrm{y}$ las niñas presentaron un menor nivel de participación en actividades físicas con una diferencia de $6,3 \%$ (Tabla 1 ).

Tabla 1. Asociación entre Nivel de actividad física en escolares

\begin{tabular}{|c|c|c|c|c|}
\hline Ítem & Calificación & Actividad & Chi cuadrado & Significancia \\
\hline $\begin{array}{l}\text { Frecuencia de actividad } \\
\text { física los últimos siete días } \\
\text { en clases educación física }\end{array}$ & Niveles buenos y moderados & Correr "muy a menudo" & $X^{2}=73,126$ & $p<0,02$ \\
\hline \multirow{2}{*}{$\begin{array}{l}\text { Actividades realizadas } \\
\text { durante el recreo }\end{array}$} & $\begin{array}{l}\text { Niños tuvieron niveles de } \\
\text { actividad física "moderados" } \\
\text { y "buenos" }\end{array}$ & $\begin{array}{l}\text { Correr o jugar fuerte mucho } \\
\text { tiempo durante el recreo }\end{array}$ & \multirow{2}{*}{$X^{2}=62,693$} & \multirow{2}{*}{$p<0,001$} \\
\hline & $\begin{array}{l}\text { Niñas tuvieron niveles de } \\
\text { actividad "moderados" }\end{array}$ & $\begin{array}{l}\text { Mantenerse paradas } \\
\text { caminando por los } \\
\text { alrededores }\end{array}$ & & \\
\hline \multicolumn{5}{|c|}{ Práctica de actividad física - Frecuencia por semana } \\
\hline 7 veces o más por semana & Niños "muy activos" & Práctica de futbol & $X^{2}=99,273$ & $p<0,001$ \\
\hline De 3 a 4 veces a la semana & $\begin{array}{l}\text { Niñas "moderadamente } \\
\text { activos" }\end{array}$ & Saltar la cuerda & $X^{2}=91,925$ & $p<0,001$ \\
\hline
\end{tabular}

$X^{2}$ chi cuadrado; $p<0,05$

La variable edad en escolares, no presentó relación con los niveles de actividad física. Independientemente de la edad los escolares se encuentran en niveles buenos y moderados de actividad física $(78,5 \%)(p<0,272)$. Para el IMC la mayoría de la población presenta un IMC ideal $(71,8 \%) \quad(p<0,272)$ y el nivel de actividad física moderado se evidencia una asociación con el género $(p<0,004)$ (Tabla 2).

Tabla 2. Niveles de actividad física según variables

\begin{tabular}{llcr}
\hline $\begin{array}{c}\text { Nivel de actividad } \\
\text { física }\end{array}$ & Variables & $\boldsymbol{X}^{\mathbf{2}}$ & \multicolumn{1}{c}{ Significancia } \\
\hline Bueno y moderado & Edad & 7,559 & 0,272 \\
Ideal & IMC & 16,95 & 0,049 \\
Moderado & Genero & 13,551 & 0,004 \\
\hline$X^{2}$
\end{tabular}

$X^{2}$ chi cuadrado; $p<0,05$
La relación entre la actividad física y el gasto frente al computador presenta asociación $(p<0,049)$. Se destaca que la mayoría de los escolares independiente del género afirmaron estar más tiempo frente al computador $(81,5 \%)$. Independientemente de la afirmación o negación, los niveles de actividad física que se evidenciaron con más frecuencia fueron entre bajo, moderado y bueno (Tabla 3).

Por otro lado, se observa que las horas diarias frente al computador juegan un papel importante. Los escolares que afirmaron utilizar el computador de 0 a 3 horas diarias se encuentran en un nivel de actividad física baja a buena, predominando la actividad física moderada con un $52,5 \%$ de la población (231 escolares), dando como resultado una significancia entre la actividad física moderada y las horas dedicadas al uso del computador $(p<0,001)$ (Figura 1). 
Tabla 3. Asociación entre actividad física y gasto frente al computador, televisión y video juegos

\begin{tabular}{|c|c|c|c|c|c|c|c|}
\hline \multirow{3}{*}{ Nivel de actividad física } & \multicolumn{7}{|c|}{ Gasto frente al computador } \\
\hline & \multicolumn{2}{|l|}{ No } & \multicolumn{2}{|c|}{$\mathbf{S i}$} & \multirow{2}{*}{$X^{2}$} & \multirow{2}{*}{ Sig } & \multirow{2}{*}{$\begin{array}{c}\text { Coeficiente de } \\
\text { contingencia }\end{array}$} \\
\hline & Frecuencia & $\%$ & Frecuencia & $\%$ & & & \\
\hline Baja & 16 & 3,62 & 51 & 11,5 & \multirow{4}{*}{3,379} & \multirow{4}{*}{0,049} & \multirow{4}{*}{0,087} \\
\hline Moderada & 43 & 9,7 & 196 & 44,3 & & & \\
\hline Buena & 22 & 4,98 & 85 & 19,2 & & & \\
\hline Muy buena & 9 & 2,0 & 20 & 4,5 & & & \\
\hline \multirow[t]{2}{*}{ Total } & 90 & 20.4 & 352 & 79.6 & & & \\
\hline & \multicolumn{7}{|c|}{ Gasto frente a la televisión } \\
\hline \multirow[t]{2}{*}{ Nivel de actividad física } & No & & \multicolumn{2}{|c|}{$\mathbf{S i}$} & \multirow{2}{*}{$X^{2}$} & \multirow{2}{*}{ Sig } & \multirow{2}{*}{$\begin{array}{c}\text { Coeficiente de } \\
\text { contingencia }\end{array}$} \\
\hline & Frecuencia & $\%$ & Frecuencia & $\%$ & & & \\
\hline Baja & 11 & 16,4 & 56 & 83,6 & & & \\
\hline Moderada & 10 & 4,2 & 229 & 95,8 & & & \\
\hline Buena & 2 & 1,9 & 105 & 98,1 & 19,339 & 0,000 & 0,372 \\
\hline Muy buena & 1 & 3,4 & 28 & 96 & & & \\
\hline \multirow[t]{2}{*}{ Total } & 24 & 5,4 & 418 & 94,6 & & & \\
\hline & \multicolumn{7}{|c|}{ Gasto frente a video juegos } \\
\hline \multirow[t]{2}{*}{ Nivel de actividad física } & No & & $\mathbf{S i}$ & & \multirow{2}{*}{$X^{2}$} & \multirow{2}{*}{ Sig } & \multirow{2}{*}{$\begin{array}{c}\text { Coeficiente de } \\
\text { contingencia }\end{array}$} \\
\hline & Frecuencia & $\%$ & Frecuencia & $\%$ & & & \\
\hline Baja & 59 & 88,0 & 8 & 12,0 & \multirow{4}{*}{59,073} & \multirow{4}{*}{0,000} & \multirow{4}{*}{0,274} \\
\hline Moderada & 151 & 63,1 & 88 & 36,9 & & & \\
\hline Buena & 33 & 30,8 & 74 & 69,2 & & & \\
\hline Muy buena & 4 & 13,7 & 25 & 86,3 & & & \\
\hline Total & 247 & 55.9 & 195 & 44.1 & & & \\
\hline
\end{tabular}

$X^{2}$ chi cuadrado; $p<0,05$

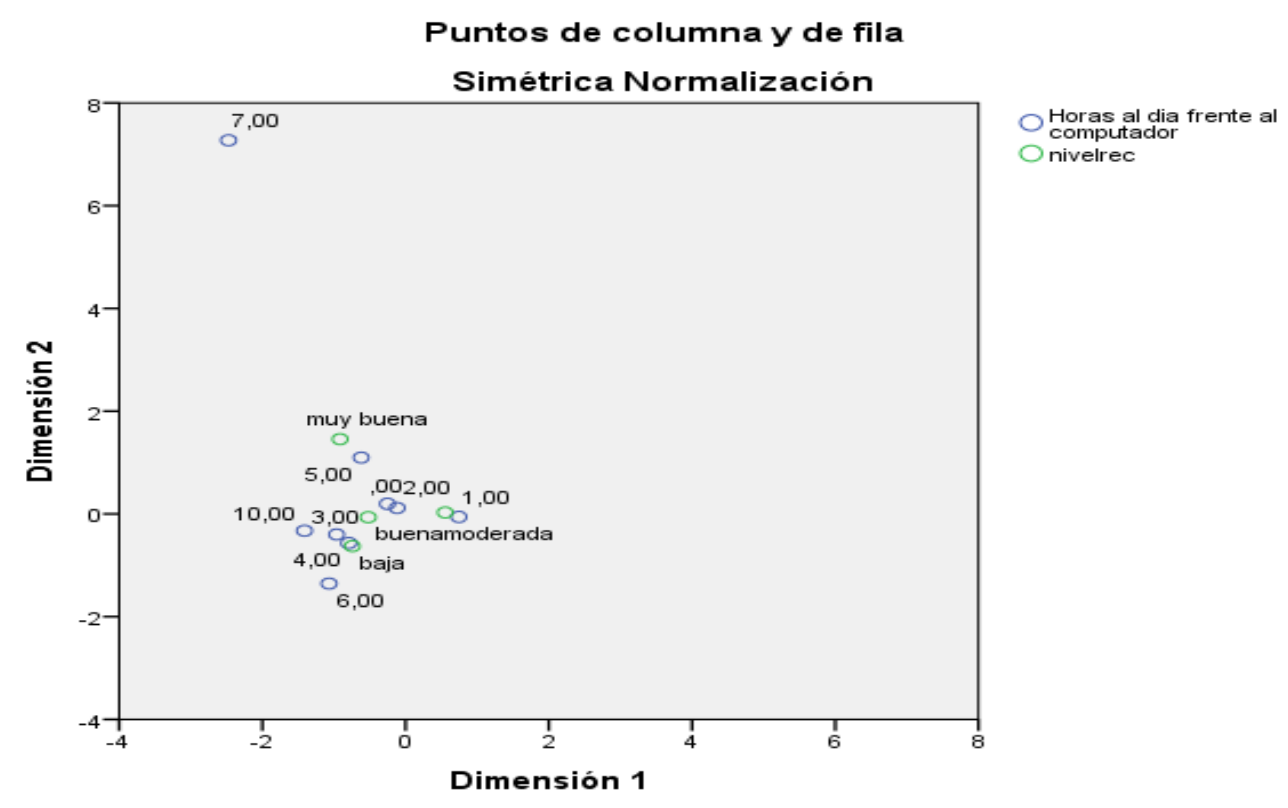

Figura 1. Análisis de correspondencia múltiple horas frente al computador y niveles de actividad física

Los escolares de todos los niveles de actividad física manifiestan ver televisión en mayor porcentaje, siendo los escolares con nivel de actividad física moderada y buena los que sobresalen (95\% y $98 \%$ respectivamente), estableciéndose que para esta relación entre la actividad física y el gasto frente al televisor existe una asociación estadísticamente significativa $(p<0,0001)$, y la fuerza de esta asociación es moderada (Tabla 3). Así mismo, los escolares que afirmaron ver entre 0 a 3 horas la televisión, se caracterizaron por niveles moderados y buenos, 
presentando una asociación entre las horas de televisión y los niveles de actividad física $(p<0,001)$.

La población escolar encuestada en mayor porcentaje niega hacer uso de videojuegos, destacándose los escolares con nivel de actividad física "moderada" (151 escolares; 63,1\%), y "baja” (59 escolares), existe una asociación estadística $(p<0,000)$ (Tabla 3). Es importante recalcar, que los niveles de actividad física general para esta población, se encuentran en el rango de niveles "moderados" en asociación con el gasto frente a la televisión (229 escolares).

La exposición de tiempo a las pantallas con relación al género evidenció que el género masculino tiene una mayor tendencia a jugar videojuegos que el género femenino. Con la información de 141 escolares femeninos que no juegan videojuegos $(31,9 \%)$, y la de 151 escolares de género masculino que afirman jugar videojuegos entre 1 y 9 horas al día $(34,1 \%)$ se sustenta la asociación entre el género y uso de videojuegos $\left(X^{2}=56,658 ; p<0,001\right)$.

Por otra parte, con respecto a la utilización del computador o del televisor, se encontró asociación con el género $\left(X^{2}=55,044 ; \quad p<0,027\right)$. Independientemente del género, la población en su gran mayoría afirmó utilizar el computador $(79,6 \%)$, con un promedio de 1 a 6 horas y estar frente al televisor $(76,2 \%)$, en un promedio de 1 a 7 horas. Los niños de 12 años estuvieron más tiempo frente al computador que sus pares de 10 y 11 años $\left(X^{2}=27,241 ; p<0,018\right)$; así mismo sucedió con el número de días frente al televisor que varió entre $1 \mathrm{y}$ 7 días $\left(\left(X^{2}=31,963\right) ;(p<0,04)\right)$. No se encontró asociación entre la edad y el gasto frente a video juegos (Tabla 4).

Tabla 4. Resumen asociación entre gasto frente a computador, televisión y video juegos y edad y sexo

\begin{tabular}{|c|c|c|}
\hline Gasto frente a computador & $\mathrm{X}^{2}$ & Sig \\
\hline Sexo & 55,044 & $0,027^{*}$ \\
\hline Edad & 31,963 & $0,04^{*}$ \\
\hline Gasto frente a televisión & $\mathrm{X}^{2}$ & Sig \\
\hline Sexo & 60,46 & $0,000^{*}$ \\
\hline Edad & 31,963 & * \\
\hline Gasto frente a video juegos & $\mathrm{X}^{2}$ & Sig \\
\hline Sexo & 56,658 & 0,001 \\
\hline Edad & 8,793 & 0,087 \\
\hline
\end{tabular}

$X^{2}$ chi cuadrado; $p<0,05$

\section{Discusión}

Los principales hallazgos del presente estudio, demuestran que existe gran asociación entre los niveles de actividad física y el gasto frente a pantallas, entendiéndose el uso de elementos tecnológicos como estilo de vida de la población escolar; aspecto que cobra gran importancia y se convierte en factor de riesgo frente a la aparición de enfermedades crónicas no transmisibles por la frecuencia de inactividad física de los escolares cuando hacen uso de estos.

Los niveles de actividad física según género corroboraron lo encontrado en otros estudios(16,17), se destaca al género masculino como el que despliega más actividades físicas; siendo éste género el que participa más activamente en actividades como el futbol, lo que se explica por la asignación de roles diferenciados a la mujer y al hombre en las actividades deportivas, de tal manera que el futbol desde un primer momento se consideró como un deporte para hombres y no de mujeres (situación que ha venido cambiando). Además, es un deporte que ha sido muy promocionado y se ha popularizado en el ámbito nacional y mundial donde los jóvenes en sus prácticas replican jugadas y quieren emular estrellas del futbol nacional e internacional(18,19).

Cabe destacar la alta frecuencia de actividades físicas por parte del género masculino en el recreo y las clases de educación física durante la semana previa a la encuesta; situación que coincide con los hallazgos del estudio de Bastos et al.,(20) donde se registra la participación regular del sexo masculino con este mismo tipo de actividades. Se vislumbra que los niños tienen más apoyo familiar y social para practicar las actividades físicas, por lo tanto, podría ser también una explicación sobre el mayor desempeño del género masculino(19,20).

El estudio registra que el género femenino presenta niveles bajos de actividad física; un resultado que es consistente con los del estudio de Aguiar et al.,(21) que muestra que el nivel de frecuencia de la actividad física puede estar influenciado por la desaprobación de sus compañeros sobre las actividades que realizan, por la influencia de los entornos familiares sobre la asociación que hay entre actividad deportiva-roles de género, y la mayor disposición del tiempo libre para actividades de entretenimiento sedentario, como el ver la televisión(21). Así mismo, Fairclough y Lynne,(22) 
afirman que la disminución de la actividad física en la población femenina, puede deberse a los procesos de maduración biológica que las niñas adquieren a temprana edad, como lo son los cambios en la estatura, la forma del cuerpo, la composición, cambios hormonales y la aparición de características sexuales secundarias antes que los niños. Lo que aumenta el riesgo de adoptar conductas poco saludables y de experimentar problemas psicosociales, incrementando a su vez, la probabilidad de que sean menos activas físicamente en comparación al género masculino.

La población escolar presentó un IMC ideal, independientemente de la edad, género y nivel de actividad física; realidad que coincide con los resultados de un estudio realizado por Schwartz et al.,(23) donde explica que en promedio la población presentaba un IMC ideal tanto para el sexo masculino como femenino, dejando en evidencia que las variables son independientes. Una investigación realizada por Gutiérrez et al.,(19) ha identificado un mayor índice de masa corporal entre el género masculino, lo cual no ha sido impedimento para la realización de actividades físicas(19).

Las pocas horas dedicadas al uso del computador están asociadas con la actividad física en nivel moderado y bueno. Este resultado puede ser influenciado por un buen estilo de vida inculcado en los niños. La Asociación Americana de Pediatría afirma que los adolescentes que ven más de 5 horas al día de televisión tienen 5 veces más probabilidad de tener sobrepeso, comparados con los adolescentes que ven de 0 a 2 horas al día; situación que se presenta debido a las influencias mediáticas y comerciales(24). García y Marina(25), en su estudio muestran que los niños que permanecen más tiempo frente a un computador presentan niveles de actividad de bajos a moderados y a su vez se relacionan con IMC en sobrepeso y obesidad.

Al igual que sucede con el uso del computador, el tiempo dedicado a los videojuegos, presentan una relación directa con la actividad física. Los niños tienen mayor riesgo de sedentarismo en comparación con las niñas. Un dato relevante que arrojó un estudio en Transilvania, es que los niños entre 7 y 11 años expuestos al uso de los videojuegos y la televisión, tienen el riesgo de sobrepeso o de obesidad y se prevé que entre más horas estén frente a una pantalla, seguramente habrá mayor sedentarismo(26). Situación que en la modernidad, donde hay una mayor difusión de tecnologías que están al alcance de un mayor número de población, especialmente en las áreas urbanas, acrecienta los riesgos de sedentarismo(26).

Entre los niveles de actividad física y el tiempo dedicado al uso del televisor, se encuentra una relación importante, donde los niveles moderados y buenos son más frecuentes en niños que permanecen menos horas de televisión al día. Estudios realizados en Australia, Brasil, Canadá, entre otros países, confirman el hecho que los escolares que se dedican más tiempo al uso del televisor con fácil acceso en casa tenían entre 9 y 13 años, eran menos activos físicamente y presentaban porcentajes altos de grasa corporal(27).

Así mismo, un estudio, confirmó que el uso prolongado del televisor en niños presenta posibilidades de adquirir enfermedades como aumento en la presión arterial y el colesterol (LDH), convirtiéndose en factores de riesgo metabólico(28). Esto puede explicarse porque los niños consumen más bebidas azucaradas y comidas no saludables cuando ven la televisión(29). Por otra parte, un estudio realizado en Brasil por Dutra et al.,(30) en el año 2015 concluye que el hábito de ver la televisión es de influencia para los niveles de actividad física y el exceso de peso en niños; mostrando una fuerte asociación entre las variables, por ello, es importante recalcar que en la población de escolares, el 37\% presentaban índices de sobrepeso y obesidad.

\section{Conclusiones}

En la investigación se identificó que la práctica de la actividad física en niños y niñas se ve influenciada especialmente por el tiempo dedicado al uso del computador y por lo tanto repercute en bajos niveles de actividad física. Esto, puede ser debido a que en la actualidad se utiliza no solo con fines académicos, sino también con fines de ocio y recreación.

Con el cuestionario PAQ-C, se identificó una clara tendencia por parte del género masculino en la práctica de actividad física de manera frecuente (bueno y moderado), contrario al género femenino, que presentó bajos niveles de actividad física, debido a que las actividades que realizaban no generaban un gasto calórico y están más asociadas al sedentarismo. 
A pesar de que los niveles de actividad física no estuvieron dentro de una calificación excelente, el IMC de los escolares se encontraba en el peso ideal, por lo tanto, no se presentó una relación directa entre la práctica de actividad física y los niveles de IMC.

Los hallazgos encontrados en la investigación dan soporte a la necesidad de realizar estrategias para promover la actividad física y establecer reglas y estilos de vida diferentes, tanto en los colegios como en los contextos familiares, de tal manera que se establezcan pautas para erradicar la inactividad física prolongada causada por el uso de las pantallas por la población colombiana. Promover la actividad física es un requisito importante para favorecer el buen desarrollo de niñas y niños, afirmando en unos casos y creando en otros, la conciencia de su importancia (especialmente en el género femenino más afectado por sus bajos niveles de actividad física) en la disminución de los índices de morbimortalidad en la edad adulta.

Se invita a que las familias asuman un protagonismo que influencie la construcción de políticas por el sector educativo encaminadas a fomentar hábitos y estilos de vida saludable que minimicen el sedentarismo en las poblaciones infantiles, en una alianza estratégica con los programas de promoción de la salud y prevención de la enfermedad del sector salud. Para garantizar el éxito se hace necesario informar y educar más sobre la importancia de mantenerse en movimiento para el bienestar. También es perentorio incentivar nuevas investigaciones para develar los factores sociales, culturales y económicos que se constituyen en obstáculos que limitan una mayor adherencia de los jóvenes a los programas de actividad física y sigan arraigados en el uso de pantallas.

\section{Recomendaciones}

Los hallazgos encontrados en la investigación fundamentan la necesidad de realizar estrategias de promoción de la actividad física, tanto en colegios como en el contexto familiar, de tal manera que se establezcan pautas para pasar de la inactividad prolongada con uso de las pantallas a la actividad física. Lo anterior será un requisito importante en la implementación de programas que favorezcan el buen desarrollo del niño, afirmando en unos casos y creando en otros, conciencia de la importancia de la actividad física y la disminución del tiempo frente a pantallas, que repercutirán en la reducción de los índices de morbimortalidad en la edad adulta.

Se hace una invitación a que las familias asuman un protagonismo que influencie la construcción de políticas por el sector educativo encaminadas a fomentar hábitos y estilos de vida saludable que minimicen el sedentarismo en las poblaciones infantiles, en interacción con el sector salud y sus programas de promoción de la salud y prevención de la enfermedad.

Futuras investigaciones podrían contribuir al conocimiento de los factores que impiden una adherencia de los jóvenes a los programas de actividad física y su relación con el uso de pantallas.

Conflicto de intereses: Ninguno declarado por los autores.

\section{Referencias}

1. Gobierno de Colombia: Ministerio de Salud. Compromiso con los estilos de vida saludables. 2017 [citado 29 Marzo 2018]. Disponible en: https://www.minsalud.gov.co/Paginas/Compromiso-conlos-estilos-de-vida-saludables.aspx

2. García Laguna DG, García Salamanca GP, Tapiero Paipa YT, Ramos DM. Determinantes de los estilos de vida y su implicación en la salud de jóvenes universitarios. Revista Hacia la Promoción de la Salud. 2012;17(2):169-85. Disponible en: https://www.redalyc.org/articulo.oa?id=309126826012

3. Salway RE, Sebire SJ, Solomon-Moore E, Thompson JL, Jago R. Associations within school-based same-sex friendship networks of children's physical activity and sedentary behaviours: a cross-sectional network analysis. J Behavioral Nutrition and Physical Activity. 2018;15(1):18. DOI: 10.1186/s12966-018-0653-9.

4. Organización Mundial De La Salud. Recomendaciones Mundiales sobre actividad física para la salud. 2010. Disponible en: https://apps.who.int/iris/bitstream/handle/10665/44441 /9789243599977_spa.pdf?sequence=1

5. Organización Mundial De La Salud. Estrategia Mundial sobre régimen alimentario, actividad física y salud. 2017. Disponible

en: https://apps.who.int/gb/ebwha/pdf_files/WHA57/A57_R1 7-sp.pdf

6. Braguinsky J. Prevalencia de obesidad en América Latina. Rev. Anales Sts san Navarra. 2002;25(Suppl 1):109-15. Disponible en: https://recyt.fecyt.es/index.php/ASSN/article/view/5493

7. Instituto Colombiano de Bienestar Familiar. ENSIN: Encuesta Nacional de situación Nutricional. 2015. Disponible en: https://www.icbf.gov.co/bienestar/nutricion/encuestanacional-situacion-nutricional 
8. Martínez-López EJ, Hita-Contreras F, Moral-García JE, GraoCruces A, Ruiz JR, Redencillas-Peiró MT, et al. Association of low weekly physical activity and sedentary lifestyle with selperceived health, pain, and well-being in a Spanish teenage population. Science \& Sports. 2015;30(6):342-51. DOI: 10.1016/j.scispo.2015.04.007.

9. Borràs PA, Ugarriza L. Obesidad infantil: ¿nos estamos equivocando? Principales causas del problema y tendencias de investigación. Apunts Med Esport. 2013;48(178):63-8. DOI: 10.1016/j.apunts.2012.09.004.

10. Ara I, Rodríguez GV, Moreno LA, Gutin B, Casajus JA. Child obesity can be better reduced through vigorous physical activity rather than through energy intake restriction. Apunts Med Esport. 2009;44(163):111-8. Disponible en: https://www.apunts.org/en-child-obesity-can-be-betterarticulo-X1886658109286649

11. Vidarte Claros JA, Vélez Álvarez C, Sandoval Cuellar C, Alfonzo Mora ML. Actividad física: Estrategia de promoción de la salud. Hacia la promoción de la salud. 2011;16(1):20218. Disponible en: http://www.scielo.org.co/pdf/hpsal/v16n1/v16n1a14.pdf

12. González S, Sarmiento OL, Lozano Ó, Ramírez A, Grijalba C. Niveles de actividad física de la población colombiana: desigualdades por sexo y condición socioeconómica. Biomedica. 2014;34(3):447-59. DOI: 10.7705/biomedica.v34i3.2258.

13. García Cruz A, Figueroa Suárez J, Osorio CJ, Rodríguez Chavarro N, Gallo Villegas J. Asociación entre el estado nutricional y las capacidades físicas en niños de 6 a 18 años de Medellín (Colombia). An Pediatr. 2014;81(6):343-51. DOI: 10.1016/j.anpedi.2013.10.040.

14. Duque IL, Parra JH. Exposición a pantallas, sobrepeso y desacondicionamiento físico en niños y niñas. Revista Latinoamericana de Ciencias Sociales, Niñez y Juventud. 2012;10(2):971-81. Disponible en: http://biblioteca.clacso.edu.ar/Colombia/alianza-cindeumz/20140410064725/art.IvanLeonardoDuque.pdf

15. Herazo-Beltrán AY, Domínguez-Anaya R. Confiabilidad del cuestionario de actividad física en niños colombianos. Rev salud pública. 2012;14(5):802-9. Disponible en: https://www.redalyc.org/articulo.oa?id=42229127007

16. Vidarte Claros JA, Vélez Álvarez C, Arango Arenas A, Parra Sánchez JH. Composición corporal en escolares colombianos. Diferencias por edad y género. Nutr. clín. diet. hosp. 2019;39(3):154-61. Disponible en: https://medes.com/publication/148825

17. Ferreira Dutra G, Correa Kaufmann C, Borges Pretto AD, Pinto Albernaz E. Sedentary lifestyle and poor eating habits in childhood: a cohort study. Ciência \& Saúde Coletiva. 2016;21(4):1051-9. DOI: 10.1590/141381232015214.08032015.

18. Prieto-Benavides DH , Correa-Bautista JE, Ramírez-Vélez R. Niveles de actividad física, condición física y tiempo en pantallas en escolares de Bogotá, Colombia: Estudio FUPRECOL. Nutr Hosp. 2015;32(5):2184-92. DOI: 10.3305/nh.2015.32.5.9576.

19. Gutierrez AA, Williams SM, Coleman MM, Garrahy DA, Laurson RK. Physical Education and Recess Contributions to Sixth Graders Physical Activity. The Physical Educator.
2016;73(1):174-90. Disponible en: https://www.proquest.com/openview/00bc3b83d37363fb d283dfe2af3ebc5f/1?pq-origsite=gscholar\&cbl=35035

20. Bastos F, Machado Reis V, Aranha AC, Garrido ND. Relação entre atividade física e desportiva, níveis de IMC, percepções de sucesso e rendimento escolar. Motricidade. 2015;11(3):41-58. DOI: 10.6063/motricidade.3771.

21. Aguiar Greca JP, Santos Silva DA, Loch MR. Physical activity and. screen time in children and adolescentes in a médium size town in the South of Brazil. Rev paul pediatr. 2016;34(3):316-22. DOI: 10.1016/j.rppede.2016.01.001.

22. Fairclough SJ, Boddy LN, Ridgers ND, Stratton G, Cumming S. Biological maturity and primary school children's physical activity: Influence of different physical activity assessment instruments. European Journal of Sport Science. 2011;11(4):241-8. DOI: 10.1080/17461391.2010.506660.

23. Schwartz AJ, Schimer M, Santini E, Domingos A. Nível de atividade física e estado nutricional em escolares do ensino fundamental. Reb Bras Obesidade, nutrição e emagrecimento. 2013;7(40):84-9. Disponible en: http://www.rbone.com.br/index.php/rbone/article/view/ 310

24. Estar constantemente conectado: efectos nocivos del consumo mediático en los niños y adolescentes [internet page]. Academia Americana de Pediatría[update Oct 2016: quoted 25 Jul 2018]. Disponible en: https://www.healthychildren.org/Spanish/familylife/Media/Paginas/Adverse-Effects-of-TelevisionCommercials.aspx

25. García Hermoso A, Marina R. Relationship of weight status, physiscal activity and screen time with academic achievement in adolescentes. Obesity research \& clinical practice. 2017;11(1):44-50. DOI: 10.1016/j.orcp.2015.07.006.

26. Doiniţa Scurt M, Neamţu M, Scurt C. A study on the dietary and physical activity practice behaviours in children aged 1215 from urban areas. Civilization and Sport. 2015;16(1):2733. Disponible en: http://pm3.ro/pdf/59/ro/09\%20$\% 20$ scurt $\% 20 \% 20 \% 20 \% 2027-33 . p d f$

27. LeBlanc AG, Katzmarzyk PT, Barreira TV, Broyles ST, Chaput J-P, Church TS, et al. Correlates of total sedentary time and screen time in 9-11 year old children around the world: The international study of childhood obesity, lifestyle and environment. PLoS One. 2015;10(6):e0129622. DOI: 10.1371/journal.pone.0129622.

28. Department of community Nutrition, School of nutrition and food sciences, Shiraz University of medical science. Eating breakfast and snacks while television viewing are associated with some cardio metabolic risk factors among Iranian Children. Diabetes \& metabolic syndrome. 2018;12(3):23543. DOI: 10.1016/j.dsx.2017.09.008.

29. Larson NI, Miller JM, Watts AW, Story MT, Neumark-Sztainer DR. Adolescent Snacking Behaviors Are Associated with Dietary Intake and Weight Status. The Journal of Nutrition. 2016;146(7):1348-55. DOI: 10.3945/jn.116.230334.

30. Dutra GF, Kaufmann CC, Pretto AD. Albernaz EP. Hábito de assistir a televisão e sua influência sobre a atividade física e o excesso de peso infantis. Jornal de Pediatria. 2015;91(4):346-51. DOI: 10.1016/j.jped.2014.11.002. 